\title{
HORMÔNIOS SEXUAIS ESTRÓGENOS: CONTAMINANTES BIOATIVOS
}

\author{
Ricardo Wagner Reis Filho* \\ Escola de Engenharia de São Carlos, Universidade de São Paulo, CP 292, 13560-790 São Carlos - SP, Brasil \\ Juliana Coutinho de Araújo e Eny Maria Vieira \\ Instituto de Química de São Carlos, Universidade de São Paulo, CP 780, 13560-970 São Carlos - SP, Brasil
}

Recebido em 3/3/05; aceito em 7/10/05; publicado na web em 12/4/06

\begin{abstract}
SEXUAL ESTROGENIC HORMONES: BIOACTIVE CONTAMINANTS. Natural and synthetic estrogens have been detected in rivers, lakes and estuaries in several parts of the world. The primary sources of these compounds are the industrial and household effluents, which are not eliminated by the received treatment. This paper presents a brief description of the problem as well as the physical and chemical characteristics of the main compounds, the environmental behavior, methods of determination, ecotoxicological aspects and a discussion about its relevance in terms of ecology and public health.
\end{abstract}

Keywords: hormones; endocrine disrupters, environmental chemistry.

\section{CONTEXTUALIZAÇÃo}

Um dos campos mais proeminentes da química ambiental é o estudo de micropoluentes orgânicos em ambientes aquáticos. Micropoluentes orgânicos são substâncias que mesmo estando presentes em pequenas concentrações, são capazes de desencadear efeitos sobre os sistemas em que são introduzidos. O termo vem sendo empregado há muito tempo tanto que Mackay $^{1}$, em 1982, já classificava como micropoluentes os compostos químicos usualmente detectados em concentrações abaixo de 1 parte por milhão $(1 \mathrm{mg}$ $\left.\mathrm{L}^{-1}\right)$. Porém, dentro deste grande grupo que virtualmente compreende um universo de milhares de compostos, os chamados disruptores endócrinos vêm se destacando em importância.

A USEPA ("United States Environmental Protection Agency") define disruptores endócrinos (EDCs) como agentes exógenos que interferem na síntese, secreção, transporte, recepção, ação, ou eliminação dos hormônios naturais do corpo, que são responsáveis pela manutenção da homeostase (preservação da constância interna), reprodução, desenvolvimento e comportamento. A Comunidade Européia ${ }^{3}$ estende os efeitos adversos dos EDCs à prole dos organismos expostos. A ação dos EDCs dá-se pelo bloqueio, pela mimetização, estimulação, ou inibição da produção dos hormônios naturais ${ }^{4}$. Estes compostos são amplamente utilizados pela sociedade moderna ${ }^{5}$, sendo encontrados em produtos farmacêuticos, produtos de uso pessoal (como ex. as fragrâncias), pesticidas, antioxidantes, plásticos, produtos industrializados, tensoativos entre outros.

A primeira hipótese sobre os efeitos dos EDCs foi levantada na década de 1980, com a observação de características femininas em machos de aves coloniais da região dos Grandes Lagos (EUA-Canadá) expostos a agrotóxicos, sendo o mesmo fenômeno relatado em populações de jacarés de lagos da Flórida ${ }^{6}$. Mas somente na década de 90 a questão emergiu como sendo uma das principais no campo da pesquisa ambiental moderna ${ }^{7}$, existindo uma intensa produção na área ${ }^{8}$ com tendência de crescimento ainda maior, devido à abrangência dos tópicos e desafios relacionados com o tema.

\section{O SISTEMA ENDÓCRINO E OS HORMÔNIOS ESTRÓGENOS NATURAIS E SINTÉTICOS}

O sistema endócrino é um mecanismo complexo que coordena e regula a comunicação entre as células, constituído por combinações de glândulas e hormônios, sendo responsável pelas funções biológicas normais, como reprodução, desenvolvimento embrionário, crescimento e metabolismo 9 . Hormônios são mensageiros químicos que respondem pela comunicação entre diferentes tipos de células, as quais identificam os hormônios através de receptores que são estruturas protéicas especializadas em reconhecimento molecular ${ }^{10}$. Depois da aproximação e interação (hormônio-receptor) ocorre uma série de reações bioquímicas, levando a respostas biológicas específicas.

Os hormônios sexuais são produzidos a partir do colesterol e podem ser classificados em três grupos principais: hormônios sexuais femininos, ou estrógenos; hormônios sexuais masculinos, ou andrógenos e, hormônios da gravidez, ou progestógenos ${ }^{11}$.

Dentre os hormônios sexuais, os estrógenos vêm recebendo maior atenção por serem compostos extremamente ativos biologicamente e estão relacionados à etiologia de vários tipos de cânceres $^{12}$. Os estrógenos naturais $17 \beta$-estradiol $\left(\mathrm{E}_{2}\right)$, estriol $\left(\mathrm{E}_{3}\right)$, estrona $\left(\mathrm{E}_{1}\right)$ e o sintético $17 \alpha$-etinilestradiol $\left(\mathrm{EE}_{2}\right)$, desenvolvido para uso médico em terapias de reposição e métodos contraceptivos, são os que despertam maior preocupação, tanto pela potência como pela quantidade contínua introduzida no ambiente. Estes hormônios possuem a melhor conformação reconhecida pelos receptores e, portanto, resultam em respostas máximas, sendo considerados como responsáveis pela maioria dos efeitos disruptores desencadeados pela disposição de efluentes ${ }^{13}$. A Figura 1 mostra a estrutura química destes compostos, sendo que algumas de suas características importantes estão sintetizadas na Tabela 1 .

Os hormônios excretados através da urina e fezes seguem para a rede coletora, adentrando depois ao ambiente. O lançamento de efluentes in natura ou mesmo processados são as principais vias de contaminação do ambiente aquático, seja pelo déficit de infra-estrutura em saneamento, seja pela ineficiência (tecnológica e/ou operacional) das estações de tratamento ${ }^{15}$. Apesar de possuírem meia-vida relativamente curta quando comparados a outros compostos orgânicos (como alguns pesticidas), os estrógenos naturais são continua-

*e-mail: reisfo@yahoo.com.br 
Tabela 1. Características dos principais estrógenos. Adaptada das ref. 8 e 14

\begin{tabular}{lccccccc}
\hline Nome comum & CAS-no & Fórmula & $\begin{array}{c}\gamma_{\text {sat }} \\
\left(\mu \mathrm{L} \mathrm{L}^{-1} 25{ }^{\circ} \mathrm{C}\right)\end{array}$ & Log $\mathrm{K}_{\text {ow }}$ & $\begin{array}{c}\text { Pressão de Vapor } \\
(\mathrm{mm} \mathrm{Hg})\end{array}$ & $\begin{array}{c}\mathrm{K}_{\mathrm{oc}} \\
(\text { dias })\end{array}$ \\
\hline $17 \beta$-Estradiol & $50-28-2$ & $\mathrm{C}_{18} \mathrm{H}_{24} \mathrm{O}_{2}$ & 12960 & 4,01 & $2,3 \times 10^{-10}$ & 3300 & $2-3 ; 0,2-9$ \\
Estrona & $53-16-7$ & $\mathrm{C}_{18} \mathrm{H}_{22} \mathrm{O}_{2}$ & 12420 & 3,13 & $2,3 \times 10^{-10}$ & 4882 & $2-3$ \\
Estriol & $50-27-1$ & $\mathrm{C}_{18} \mathrm{H}_{24} \mathrm{O}_{3}$ & 13250 & 2,45 & $6,7 \times 10^{-15}$ & 1944 & $\mathrm{NR}$ \\
$17 \alpha$-Etinilestradiol & $57-63-6$ & $\mathrm{C}_{20} \mathrm{H}_{24} \mathrm{O}_{2}$ & 483 & 3,67 & $4,5 \times 10^{-11}$ & 4770 \\
\hline
\end{tabular}

$\gamma_{\text {sat }}$ : solubilidade em água; $\mathrm{K}_{\mathrm{ow}}$ : coeficiente de partição octanol/água; $\mathrm{K}_{\mathrm{oc}}$ : constante de sorção; NR: não relatado.

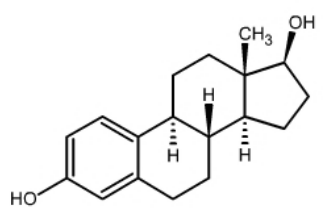

$17 ß-$ Estradio

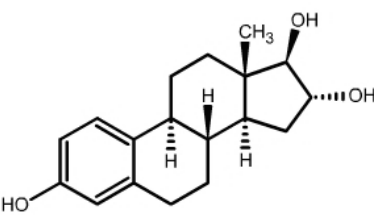

Estriol

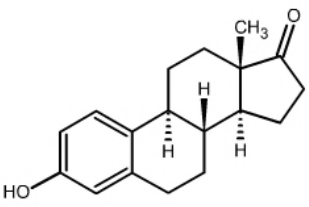

Estrona

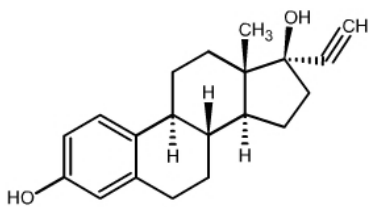

$17 \alpha-$ Etinilestradio
Figura 1. Estruturas dos principais hormônios estrógenos

mente introduzidos no ambiente (Tabela 2), o que lhes concede um caráter de persistência. Estudos relatam que até $40 \%$ das doses ministradas de estrógenos sintéticos podem ser disponibilizadas para o ambiente $^{16}$. A Figura 2 exemplifica o modo de entrada destes contaminantes para os ecossistemas. Embora grande parte dos estrógenos seja metabolizada e excretada na forma inativa, conjugada como glicuronídeos e sulfatos ${ }^{17}$, a ação de enzimas produzidas por bactérias comumente encontradas em áreas de despejo de efluentes prontamente os biotransformam em compostos biologicamente ativos e passíveis de desencadearem efeitos deletérios ${ }^{18}$.

$\mathrm{O}$ destino dos estrógenos no ambiente depende de suas características físicas e químicas e das propriedades do meio receptor. As inúmeras variáveis que atuam em conjunto no ambiente aquático, como temperatura, turbidez, $\mathrm{pH}$, alcalinidade, oxigênio dissolvido, radiação, matéria orgânica e concentração de diversas outras substâncias, tornam a tarefa de modelar o comportamento destes compostos bastante complexa. Devido a sua lipofilicidade e baixa volatilidade, o processo de sorção em sedimentos suspensos pode ser um fator significativo na redução dos estrógenos na fase aquo$\mathrm{sa}^{19}$. Porém, em estudos de partição entre sedimento e água simulan- do várias condições ambientais, Bowman et al. ${ }^{20}$ concluíram que embora as partículas influenciem no comportamento ambiental dos estrógenos, a sorção é relativamente limitada, com a maioria permanecendo na fase aquosa. Já Lai et al. ${ }^{19}$ sugeriram que estrógenos dissolvidos podem rapidamente se tornar adsorvidos aos sólidos em suspensão, indicando que a competição pelos sítios de ligação com outros compostos mais hidrofóbicos e a respectiva saturação destes sítios são os responsáveis pela proporção de estrógenos que permanecem na fase aquosa. São necessárias investigações mais detalhadas para se entender a dinâmica de distribuição dos hormônios nos diversos ambientes em que são inseridos.

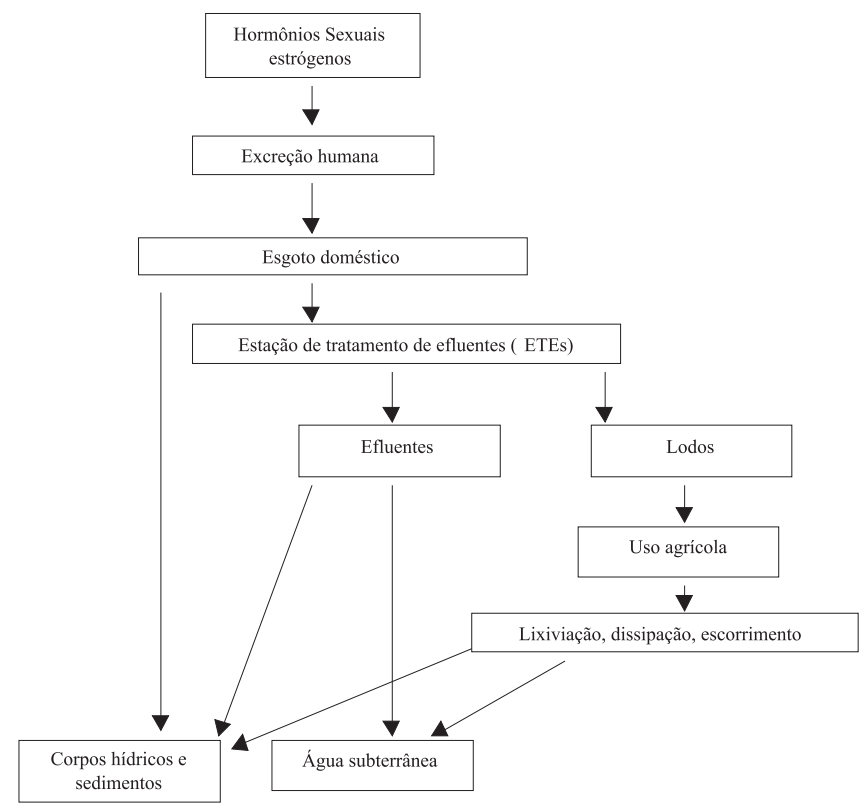

Figura 2. Representação esquemática da principal via de entrada de disruptores endócrinos hormonais em sistemas aquáticos. Adaptada da ref. 22

\section{TÉCNICAS ANALÍTICAS}

A análise em nível de traços de compostos orgânicos em água pode ser considerada como um dos maiores desafios da química

Tabela 2. Quantidade média de estrógenos diariamente excretada na urina de humanos. Adaptada da ref. 21

\begin{tabular}{|c|c|c|c|c|}
\hline Estrógeno & $\begin{array}{c}\text { Excreção } \hat{\delta} \\
(\mu \mathrm{g} / 24 \mathrm{~h})\end{array}$ & $\begin{array}{c}\text { Excreção }+ \\
\text { menstruação } \\
(\mu \mathrm{g} / 24 \mathrm{~h})\end{array}$ & $\begin{array}{c}\text { Excreção } 9 \\
\text { gravidez } \\
(\mu \mathrm{g} / 24 \mathrm{~h})\end{array}$ & $\begin{array}{c}\text { Excreção } 9 \\
\text { menopausa } \\
(\mu \mathrm{g} / 24 \mathrm{~h})\end{array}$ \\
\hline $17 \beta$-Estradiol & 1,6 & 3,5 & 259 & 2,3 \\
\hline Estrona & 3,9 & 8,0 & 600 & 4,0 \\
\hline Estriol & 1,5 & 4,8 & 6000 & 1,0 \\
\hline
\end{tabular}


analítica. A determinação de hormônios estrógenos no ambiente constitui-se em tarefa difícil, primeiro, devido à complexidade das matrizes ambientais e, segundo, por causa de sua baixa concentração (na ordem de $n g \mathrm{~L}^{-1}$ ), porém fisiologicamente ativa ${ }^{23}$.

Na maioria das análises que envolvem amostras "reais", é necessário o enriquecimento substancial do analito para se isolar os compostos alvos da matriz e atingir os limites de detecção e quantificação requeridos. Um procedimento analítico típico inclui, portanto, vários passos para preparação da amostra, tais como filtração, extração, purificação e evaporação. Se a determinação final for feita por cromatografia gasosa, hidrólise e derivatização também são, freqüentemente, necessárias ${ }^{24}$.

Em análises de água, até alguns anos, na etapa de preparação das amostras era comum se fazer a extração dos componentes de interesse utilizando a extração líquido-líquido. Porém, esse tipo de extração, além de ser uma técnica tediosa, requer grandes volumes de solventes orgânicos, apresenta custo elevado e difícil automação ${ }^{25}$.

Em meados da década de 70, visando a eliminação desses problemas, uma nova técnica foi introduzida, a qual tem sido denominada extração em fase sólida ("SPE: Solid Phase Extraction") ${ }^{25}$, onde grupos funcionais orgânicos hidrofóbicos são quimicamente ligados a uma superfície sólida, como sílica. Um exemplo comum é a ligação do grupo $\mathrm{C}_{18}$ com a sílica, sendo que esse grupo interage com compostos orgânicos hidrofóbicos pela ação das forças de van der Waals e, dessa forma, são extraídos da fase aquosa ${ }^{26}$. Uma alternativa ao uso de cartuchos em extração em fase sólida foi o desenvolvimento de discos constituídos de fases estacionárias cromatográficas imobilizadas com material inerte como PTFE (politetrafluoretileno) ou fibra de vidro. Os discos são preferidos quando são manipulados ${ }^{25}$ grandes volumes de amostras.

Segundo Barceló et al. ${ }^{24}$, a extração de estrógenos e progestógenos em água é feita usualmente pela extração em fase sólida (SPE) em discos, ou mais freqüentemente cartuchos, sendo o adsorvente mais amplamente empregado o octadecilsilano (C18) quimicamente ligado à sílica e, em menor escala, com sorventes poliméricos de carbono preto grafitizado (GCB).

Para determinação de EDCs, a cromatografia gasosa acoplada à espectrometria de massas (CG-EM) tem sido a técnica mais comumente empregada, uma vez que apenas há poucos anos a cromatografia líquida acoplada à espectrometria de massas (CLEM) tem ganhado popularidade ${ }^{27}$.

O pré-requisito necessário para análise em cromatografia gasosa é que o analito de interesse seja volátil e termicamente estável. Quando não for o caso, a derivatização pode ser usada para superar esta limitação. Tradicionalmente, a cromatografia gasosa necessita do uso de derivados para determinar compostos estrogênicos. As desvantagens da derivatização são o trabalho intensivo de laboratório e a possibilidade de redução da recuperação do analito, pois a hidrólise dos conjugados para estrógenos livres, via derivatização, pode compor erros nos estágios de recuperação, extração e quantificação, devido à baixa eficiência no passo de hidrólise ${ }^{28}$. O tempo de consumo na etapa de derivatização e a possível perda do analito têm levado a considerar a técnica de cromatografia líquida como preferida para determinação de estrógenos.

A cromatografia líquida tem várias vantagens para análise de compostos orgânicos em água. Uma delas é que os compostos voláteis representam uma pequena fração de compostos orgânicos contidos em água e esgotos. A maior parte do carbono está presente como compostos não voláteis, que podem ser diretamente analisados pela cromatografia líquida e não pela gasosa. Isto é especialmente verdadeiro para esgotos, os quais contêm muito material húmico e compostos orgânicos polares, tais como carboidratos ${ }^{29}$.
De acordo com vários autores, os detectores e acoplamentos de detectores mais empregados para análise de hormônios pela técnica de CLAE são: eletroquímico ${ }^{30,31}$, fluorescência ${ }^{32}$, espectrômetro de massas ${ }^{24,27,28,31,33}$ - CLAE-EM, CLAE-EM-EM, ultravioleta com varredura de diodo ${ }^{23}$ e ultravioleta com varredura de diodo acoplado a espectrômetro de massas ${ }^{23,34}$.

\section{TOXICIDADE}

Os organismos podem ser considerados como um imenso complexo de reações químicas ininterruptas, desde o zigoto, o embrião, o feto, a fase jovem e, finalmente, a idade adulta ${ }^{35}$. Durante esta evolução, as reações são constantemente alteradas à medida que inúmeros agentes químicos adentram ao sistema, como alimentos, bebidas, medicamentos, drogas, gases, etc. A Figura 3 mostra a ação dos estrógenos sobre os mecanismos de controle do sistema endócrino, desencadeando perturbações em cada um dos aspectos controlados pelo sistema, incluindo a homeostase que é fundamental para manutenção do equilíbrio metabólico dos organismos.

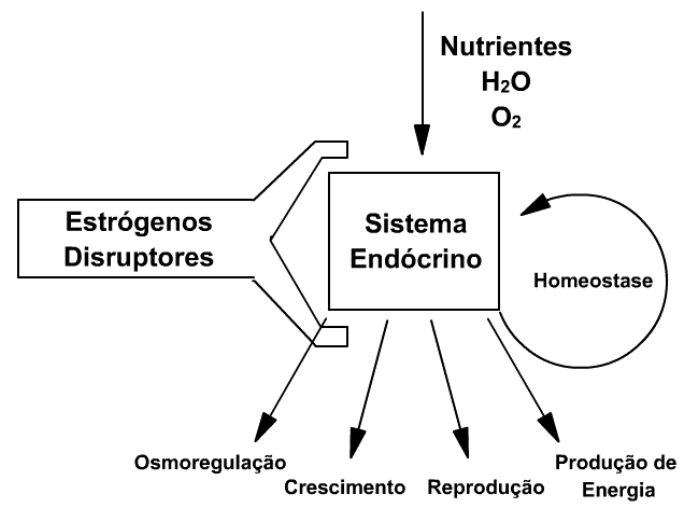

Figura 3. Representação esquemática da interação tóxica dos estrógenos com as funções coordenadas pelo sistema endócrino. Adaptada da ref. 36

\section{Ecotoxicologia}

Os efeitos desencadeados pelos hormônios dispostos no ambiente atingem desde microinvertebrados até grandes vertebrados, sendo amplamente relatados na literatura científica e considerados como uma questão de âmbito global ${ }^{37}$. Agências ambientais de diversos países, assim como organizações internacionais, desenvolvem programas e planos de pesquisa que versam sobre o tema ${ }^{38}$.

Segundo Manahan ${ }^{39}$, a principal preocupação ecotoxicológica com estas substâncias implica em sua evidente capacidade de afetar a reprodução das espécies e interferir no desenvolvimento saudável da prole. Assim, o estágio do desenvolvimento em que a exposição ocorre é particularmente importante pois, em espécies aquáticas onde a fase embrio-larval é crítica, danos permanentes podem ser provocados em vários órgãos e sistemas ${ }^{40}$.

De acordo com Erickson ${ }^{15}$, o dirigente da seção de química ambiental da USEPA, Christian Daughton, afirma que a concentração de EDCs no ambiente é apenas um dos aspectos do problema, sendo imprescindível o conhecimento da sua respectiva potência, pois muitos deles são preocupantes em qualquer concentração mensurável. Assim, as curvas de dose-resposta dos hormônios ambientais diferem das normalmente associadas a outros agentes tóxicos (como ex., metais), tendo respostas expressivas geradas em concentrações extremamente baixas ${ }^{41}$. A interpretação do conceito 
de limiar de segurança é inapropriado para este grupo de poluentes. Da Matta e Azevedo ${ }^{42}$ salientam a inexistência de consenso sobre a metodologia adequada para abordar o risco gerado por substâncias onde para o efeito crítico não é passível se estabelecer limites.

Inúmeros são os efeitos desencadeados pelos hormônios sexuais sobre a biota ${ }^{39}$ : alterações nas taxas de fecundidade, fertilização, eclosão; modificações comportamentais (agressividade, movimentação); histopatologias (fígado, gônadas, rins); imunodepressão; imposex (desenvolvimento de características sexuais femininas em machos ou oposto) e, inibição do desenvolvimento dos órgãos sexuais e reversão sexual. Os vários efeitos manifestam-se após interação entre agentes e receptores bioquímicos. Portanto, as respostas bioquímicas são perceptíveis antes que os efeitos sejam observáveis em níveis de organização superior, como em populações, comunidades e ecossistemas. Este cenário levou à busca de "sinais" prematuros de advertência, os biomarcadores ${ }^{43}$. Biomarcadores são quaisquer respostas biológicas decorrentes de reações químicas medidas em nível sub-individual, tanto dentro do organismo (enzimas, proteínas, hormônios, aminoácidos, material genético etc.) como em seus produtos (urina, fezes, pêlos etc.), indicando um desvio das condições normais não detectadas em organismos intactos ${ }^{44}$.

A determinação da proteína vitelogenina (VTG) em organismos aquáticos, principalmente peixes, tem sido bastante utilizada na investigação por contaminantes estrogênicos ${ }^{45}$. A vitelogenina é uma fosfolipoglicoproteína sintetizada por todas as fêmeas de ovíparos durante o ciclo reprodutivo; é produzida no fígado e secretada na corrente sanguínea, onde é transportada até os ovários, acumulando-se nos ovócitos em crescimento para ser, então, utilizada como precursora das reservas nutricionais necessárias para o desenvolvimento subseqüente dos embriões ${ }^{46}$. Em indivíduos imaturos ou em machos, a codificação do gene para esta proteína não existe ou é muito fracamente expressada. Assim, a presença desta proteína no sangue destes organismos representa um biomarcador de exposição, pois sua síntese depende da presença de xenoestrógenos (Figura 4).

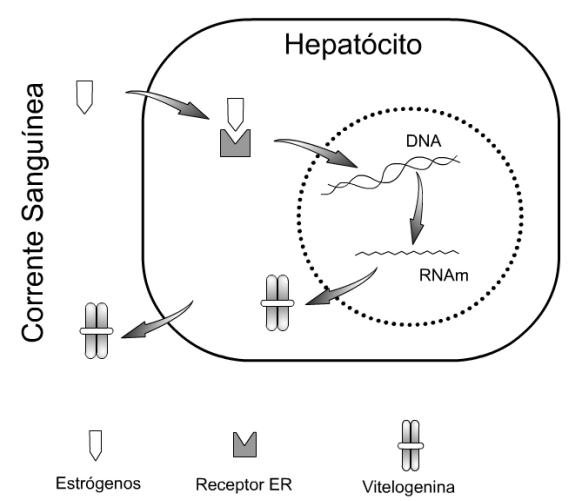

Figura 4. Representação esquemática simplificada da seqüencia de processos que leva à síntese do biomarcador de exposição VTG em peixes machos

Além de proporcionar uma avaliação qualitativa de exposição a agentes estrogênicos, a própria produção da VTG no gênero masculino envolve prejuízos, ainda que indiretos, para o desenvolvimento dos organismos. A sobrecarga das funções hepáticas e a disrupção metabólica, devido ao desvio na produção de proteínas essenciais em detrimento da produção da VTG, juntamente com a baixa na resistência imunológica e retardo no crescimento são alguns dos possíveis danos apontados na literatura ${ }^{46,47}$.

\section{TIE: testes de toxicidade e química analítica}

A avaliação e identificação da toxicidade, conhecida como TIE (“Toxicity Identification Evaluation”), é uma metodologia que combina Química e Biologia em programas de monitoramento. O TIE utiliza procedimentos de fracionamento para distinguir quais entre as várias classes de compostos que compõem um efluente ou amostra ambiental são responsáveis por gerar toxicidade ${ }^{48}$. A técnica é estruturada em três fases seqüenciais: caracterização, identificação e confirmação da toxicidade ${ }^{49}$. Diversos procedimentos são empregados para separar os constituintes responsáveis pela toxicidade ${ }^{48}$ : filtração, quelação (EDTA), aeração, extração em fase sólida (colunas $\mathrm{C}_{18}$ ) e alterações de $\mathrm{pH}$ são algumas entre as possibilidades de manipulação. Mudanças na toxicidade após estes tratamentos são indicativas da contribuição das diferentes classes de compostos.

Routledge $^{50}$ descreveu a aplicação de técnica para identificação de substâncias endócrinas ativas. Através de extração em fase sólida $\left(\mathrm{C}_{18}\right)$ e eluição seletiva, removeu compostos orgânicos de efluentes complexos e os identificou usando de técnicas cromatográficas, sendo que a atividade estrogênica avaliada por meio de ensaios com levedura in vitro indicou os hormônios sexuais estrógenos como os principais responsáveis pelos efeitos atribuídos aos efluentes domésticos. Gomes et al. ${ }^{28}$, em revisão sobre os métodos de determinação de disruptores endócrinos em amostras aquosas, indicaram uma série de trabalhos que aplicaram esta estratégia, descrevendo também uma abordagem coerente da aplicação do TIE em estudos com substâncias potencialmente estrogênicas.

\section{PERSPECTIVAS: ECOLOGIA E SAÚdE PÚBLICA}

O lançamento de substâncias hormonalmente ativas em corpos hídricos, mesmo em baixas concentrações, pode acarretar sérios impactos sobre a dinâmica e estrutura das populações aquáticas ${ }^{51}$. Estudos importantes realizados no Canadá ${ }^{52}$ indicaram, pela primeira vez, que concentrações do estrógeno $17 \alpha$-etinilestradiol, iguais as encontradas nas proximidades de lançamentos de efluentes (5-6 ng L ${ }^{-1}$ ) levaram ao colapso as populações de peixes existentes em lagos experimentais. Como nos ecossistemas existem intrincadas teias tróficas, o desequilíbrio provocado pela extinção de uma espécie-chave, por mais resiliente que seja o sistema, pode gerar mudanças significativas e/ou imprevisíveis em razão das propriedades emergentes. É importante salientar que a literatura ${ }^{53,54}$ relata concentrações de $17 \alpha$-etinilestradiol em efluentes desde 0,5 até $273 \mathrm{ng} \mathrm{L}^{-1}$, em razão do tipo de tratamento empregado e do local amostrado.

Além de seu valor intrínseco, as espécies selvagens fazem parte das estruturas e funções dos ecossistemas que suportam as atividades humanas. Aliás, ecologicamente os humanos são apenas uma entre as milhares de espécies que habitam o planeta. A postura que desvincula o Homem Natural e valoriza apenas a "tecnosfera" é a grande responsável por nossa sociedade altamente industrializada e poluídora.

Em 1987, o jornal da Associação Médica Americana (JAMA) já mencionava a prescrição de estrógenos para cerca de 200 tratamentos terapêuticos ${ }^{55}$. Mais recentemente, Weeb ${ }^{56}$ citou que em 2002 os médicos americanos prescreveram uma média de 10,6 medicamentos (de diferentes classes) para cada habitante. Sanderson et $a l . .^{57}$ demonstraram através de estudos de toxicidade que, entre as principais classes de medicamentos encontradas em amostras ambientais, os hormônios sexuais estão entre os que podem induzir efeitos mais severos, tanto para humanos quanto para a biota. 
Outro dado relevante foi levantado por Zlidar et al..$^{58}$, em que mais de 620 milhões de mulheres casadas ao redor do mundo fazem uso de métodos contraceptivos, estando as pílulas anticoncepcionais e os injetáveis entre os mais utilizados. Estes fatos evidenciam as altas taxas de uso e conseqüente introdução desses compostos no ambiente.

$\mathrm{O}$ estabelecimento de um nexo causal considerando o ambiente em doenças crônicas é uma tarefa complexa, devido às inúmeras exposições a que os seres humanos são submetidos diariamente. No entanto, questões relevantes relacionadas aos EDCs, apesar de ainda necessitarem de processos rigorosos e exaustivos de investigação científica, não podem ser subestimadas. A antecipação da puberdade $^{59}$ e o declínio na qualidade do sêmen ${ }^{60}$ durante o último século são temas centrais nesta discussão. A existência de sítios receptores para estrógenos em diversos tecidos ${ }^{61}$ e a capacidade destes compostos atuarem de modo sinérgico ${ }^{62}$ reforçam a preocupação existente com a disrupção endócrina.

Há muito tempo, uma nova postura do Homem frente ao ambiente se faz necessária, com intuito de preservar a integridade da vida. Um dos pontos fundamentais neste novo paradigma é em relação à saúde, que deixa de ser "apenas" um direito para se transformar em uma responsabilidade individual e sócio-ambiental.

\section{AGRADECIMENTOS}

À FAPESP pela concessão da bolsa de doutoramento processo 03/05772-0. Ao CNPq pela concessão de bolsa de mestrado. Ao biólogo D. S. Barbosa pelo trabalho computacional nas ilustrações.

\section{REFERÊNCIAS}

1. Mackay, D.; Water Sci. Technol. 1982, 14, 5.

2. United States Environmental Protection Agency (USEPA); Research Plan for Endocrine Disruptors, Washington, 1998

3. European Workshop on the Impact of Endocrine Disruptors on Human Health and Wildlife, Weybridge, 1996.

4. http://e.hormone.tulane.edu/learning/effects.html, acessada em Janeiro 2005.

5. Thomas, J. A.; Int. J. Toxicol. 1998, 17, 129; Jørgensen, S. E.; Sørensen, B. H.; Chemosphere 2000, 40, 691; Kolpin, D. W.; Furlong, E. T.; Meyer M. T.; Thurman, E. M.; Zaugg, S. D.; Barber, L. B.; Buxton, H. T.; Environ Sci. Technol. 2002, 36, 1202

6. Society of Environmental Toxicology and Chemistry (SETAC); Endocrine Disruptors and Modulators, Pensacola, 2000.

7. Brönmark, C.; Hansson, L. A.; Environ. Conserv. 2002, 29, 290

8. Lintelmann, J.; Katayama, A.; Kurihara, N.; Shore, L.; Wenzel, A.; Pure Appl. Chem. 2003, 75, 631.

9. http://www.ec.gc.ca, acessada em Novembro 2000; Raphael, J. W.; Regul. Toxicol. Pharmacol. 2002, 36, 118; McGovern, P.; McDonald, H. S. WE\&T 2003, 15, 35 .

10. Simmonds, R. J.; Chemistry of Biomolecules: An Introduction, The Royal Society of Chemistry: Cambridge, 1992.

11. Solomons, G.; Fryhle, C.; Química Orgânica, 7ª ed., LCT: Rio de Janeiro, 2000

12. Dos Reys, L. L.; RFML 2001, 6, 213.

13. Gray, T. P. R.; Jobling, S.; Morris, S.; Kelly, C.; Kirby, S.; Janbakhsh, A.; Harries, J. E.; Waldock, M. J.; Sumpter, J. P.; Tyler, C. R.; Environ. Sci. Technol. 2000, 34, 1521; Shaw, I.; McCully, S.; Int. J. Food Sci. Technol. 2002, 37, 471; Suzuki, K.; Hirai, H.; Murata, H.; Nishida, T.; Water Res. 2003, 37, 1972; Aerni, H. R.; Kobler, B.; Rutishauser, B. V.; Wettstein, F. E.; Fischer, R.; Giger, W.; Hungerbühler, A.; Marazuela, M.D.; Peter, A.; Schönenberger, R.; Vögeli, A.C.; Suter, M.J.F.; Eggen, R. I. L.; Anal. Bioanal. Chem. 2004, 378, 688 .

14. Tabak, H. H.; Blomhuff, R. N.; Bunch, R. L.; Dev. Ind. Microbiol. 1981 22, 497; Jürgens, M. D.; Holthaus, K. I. E.; Johnson, A. C.; Smith, J. J. L.; Environ. Toxicol. Chem. 2002, 21, 480; Carballa, M.; Omil, F.; Lema, J. M.; Llompart, M.; Jares, C. G.; Rodríguez, I.; Gómez, M.; Ternes, T.; Water Res. 2004, 38, 2918.

15. Erickson, B. E.; Environ. Sci. Technol. 2002, 36, 141A.
16. Johnson, A. C.; Williams, R. J.; Environ. Sci. Technol. 2004, 38, 3649

17. Arie, W. M. Y.; Arie, M. H. A.; Da Fonseca, A. M.; Halbe, H. W.; Bagnoli, V. R. Em Estrogênios em Ginecologia Endócrina Manual de Normas; Da Fonseca, A. M.; Bagnoli, V. R.; Halbe, H. W.; Pinotti, J. A., eds.; Roca: São Paulo, 2004

18. Tyler, C. R.; Routledge, E. J.; Pure Appl. Chem. 1998, 70, 1795; Panter, G. H.; Thompson, R. S.; Beresford, N.; Sumpter, J. P.; Chemosphere 1999, 38,3579 .

19. Lai, K. M.; Johnson, K. L.; Scrimshaw, M. D.; Lester, J. N.; Environ. Sci. Technol. 2000, 34, 3890 .

20. Bowman, J. C.; Readman, J. W.; Zhou, J. L.; Environ. Geochem. Health 2003, 25, 63

21. Johnson, A. C.; Belfroid, A.; Di Corcia, A.; Environ. Sci. Technol. 2000, $34,163$.

22. Velagaletti, R. R.; Drug Infor. J. 1995, 250, 565.

23. Barceló, D.; Alda, M. J. L.; J. Chromatogr., A 2001, 911, 203.

24. Barceló, D.; Alda, M. J .L.; Díaz-cruz, S.; J. Chromatogr., A 2003, 1000, 503.

25. Lanças, F .M.; Extração em Fase Sólida (SPE), Rima: São Carlos, 2004.

26. Christian, G .D.; Analytical Chemistry, Wiley: New York, 1994.

27. Ingrand, V.; Herry, G.; Beausse, J.; Roubin, M.; J. Chromatogr., A 2003 , 1020, 99.

28. Gomes, L. R.; Scrimshaw, D. M.; Lester, J. N.; Trends Anal. Chem. 2003, 22, 697

29. Grob, R. L.; Chromatographic Analysis of the Environment, $2^{\text {nd }}$ ed., Dekker: New York, 1983.

30. Shimada, K.; Tanaka, T.; Nambara, T.; J. Chromatogr. B: Anal. Technol. Biomed. Life Sci. 1981, 223, 33; Reid, J .J.; Stitzel, R. E.; Head, R. J.; J. Pharmacol. Methods 1985, 14, 25; Shimada, K.; Nagashima, E.; Ori, I.; Nambara, T.; J. Pharmacol. Biomed. Anal. 1987, 5, 361; Fernández, N. J. J.; García, M. J.; Diez, M. T.; J. Chromatogr. Biomed. Applic. 1993, 619, 143.

31. Giese, R. W.; J. Chromatogr., A 2003, 1000, 401.

32. Gatti, R.; Gotti, R.; Gioia, M. G.; Cavrini, V.; J. Pharmacol. Biomed. Anal. 1998, 17, 337; Gatti, R.; Gioia, M. G.; Di Pietra, A. M.; Cavrini, V.; J. Pharmacol. Biomed. Anal. 1998, 18, 187; Novakovi, J.; Tvrzická, E.; Pacáková, V.; J. Chromatogr., A 1994, 678, 359; Matsumoto, K.; Tsukahara, Y.; Uemura, T.; Tsunoda, K.; Kume, H.; Kawasaki, S.; Tadano, J.; Matsuya, T.; J. Chromatogr., B: Anal. Technol. Biomed. Life Sci. 2002, 773, 135; De Boer, T.; Otjens, D.; Muntendamb, A.; Meulmanb, E.; van Oostijen, M.; Ensing, K.; J. Pharmacol. Biomed. Anal. 2004, 34, 671; Mao, L.; Sun, C.; Zhang, H.; Li, Y.; Wu, D.; Anal. Chim. Acta 2004, 522, 241.

33. Isobe, T.; Shiraishi, H.; Yasuba, M.; Shinoba, A.; J. Chromatogr., A 2003, 984, 195; Brossa, L.; Pocurull, E.; Borrull, F.; Marcé, R. M.; Chromatographia 2004, 59, 419; Làgana, A.; Bacaloni, A.; Leva, I.; Faberi, A.; Fago, G.; Marino, A.; Anal. Chim. Acta 2004, 501, 79.

34. Barceló, D.; Alda, M. J. L.; J. Chromatogr., A 2001, 938, 148.

35. Corwin, H.; Leo, A.; Exploring QSAR: Fundamentals and Applications in Chemistry and Biology, ACS Professional Reference Book: Washington, 1999.

36. Brouwer, A.; Murk, A. J.; Koeman, J. H.; Func. Ecology 1990, 4, 275.

37. International Programe on Chemical Safety (IPCS); Global Assessment of the State-of-Science of Endocrine Disruptors, Geneva, 2002.

38. http://www.nihs.go.jp/hse/endocrine/index.htm, acessada em Outubro 2001; http://www.who.int/pcs/, acessada em Março 2003; http://endocrine.ei.jrc.it, acessada em Março 2003; http://oecd.org/ehs/endocrin.htm, acessada em Maio 2003.

39. Manahan, S.E.; Toxicological Chemistry and Biochemistry, $3^{\text {rd }}$ ed., Lewis Publishers: Boca Raton, 2003.

40. Medical Research Counci'ls Institute for Environment and Health (MRC); Appraisal of Test Methods for Sex-Hormone Disrupting Chemicals Capable of Affecting the Reproductive Process, London, 1998.

41. Lathers, C. M.; J. Clin. Pharmacol. 2002, 42, 7.

42. Da Matta, C. A. A.; Azevedo, F. A. Em As Bases Toxicológicas da Ecotoxicologia; Da Matta, C. A. A.; Azevedo, F. A., coord.; Rima: São Carlos/Intertox: São Paulo, 2003, cap. 5.

43. van der Oost, R.; Beyer, J.; Vermeulen, N. P. E.; Environ. Toxicol. Pharmacol. 2003, 13, 57.

44. Adams, W. J. Em Aquatic Toxicology Testing Methods in Handbook of Ecotoxicology; Hoffman, D. J.; Rattner, B. A.; Burton Jr., G. A.; Cairnas Jr., J., eds.; Lewis Publishers: Boca Raton, 1995, cap. 3; van Gestel, C. A. M.; van Brummelen, T. C.; Ecotoxicology 1996, 5, 217.

45. Sumpter, J. P.; Jobling, S.; Environ. Hlth. Perspect. 1995, 103,173; Tyler, C. R.; van der Eerden, B.; Jobling, S.; Panter, G.; Sumpter, J. P.; J. Comp. Physiol. B 1996, 166, 418; Hansen, P. D.; Dizer, H.; Hock, B.; Marx, A.; Sherry, J.; McMaster, M.; Blaise, C.; Trends Anal. Chem. 1998, 17, 448; 
Allner, B.; Wegener, G.; Knacker, T.; Allner, P. S.; Sci. Total Environ. 1999, 233, 21; Takemura, A.; Kim, B. H.; Comp. Biochem. Physiol., Part A: Mol. Integr. Physiol. 2001, 129, 641; http://www.comparative-hepatology.com/ content/2/1/4, acessada em Fevereiro 2005.

46. Institut National de L'environnement Industriel et des Risques (INERIS); Annual and Scientific Report, Paris, 2000.

47. Solé, M.; Castillo, M.; Alda, M. J. L.; Porte, C.; Barceló, D.; Analusis 2000, $28,783$.

48. Swartz, R. C.; Di Toro, D. M. Em Sediments as Complex Mixtures: An Overview of Methods to Assess Ecotoxicological Significance in Ecological Risk Assessment of Contaminated Sediments; Ingersoll, C. G.; Dillon, T.; Biddinger, G. R., eds.; Setac Press: Pensacola, 1997, cap. 16.

49. Ankley, G. T.; Berigan, S. M. K.; J. Aqua. Ecosys. Hlth. 1995, 4, 133.

50. Routledge, J. E.; Pure Appl. Chem. 2003, 75, 2461.

51. Folmar, L. C.; Hemmer, M.; Hemmer, R.; Bowman, C.; Kroll, K.; Denslow, N. D.; Aquat. Toxicol. 2000, 49, 77.

52. Pelley, J.; Environ. Sci. Technol. 2003, 37, 313A

53. Bila, D. M.; Dezotti, M.; Quim. Nova 2003, 26, 523.
54. Nash, J. P.; Kime, D. E.; van der Ven, L. T. M.; Wester, P. W.; Brion, F.; Maack, G.; Allner, P. S.; Tyler, C. R.; Environ. Hlth. Perspect. 2004, 112, 1725

55. Palmlund, I.; J. Psychosom. Obest. Gynoc. 1996, 17, 71.

56. Weeb, C. E.; IEEE Spectrum 2004, 41, 37.

57. Sanderson, H.; Brain, R. A.; Johnson, D. J.; Wilson, C. J.; Solomon, K. R.; Toxicology 2004, 203, 27.

58. http://www.populationreports.org/m17/, acessada em Novembro 2003.

59. Pike, M. C.; Ross, R. K.; Br. Med. Bull. 1984, 40, 351; Multigner, L.; Oliva, A.; Cad. Saúde Pública 2002, 18, 403.

60. Altken, R. J.; Koopman, P.; Lewis, S. E. M.; Nature 2004, 432, 48.

61. Mueller, S. O.; Anal. Bioanal. Chem. 2004, 378, 582; Jacobs, M. Em Pesticide Action Network UK 2001, (briefing 1), 1; Shughrue, P. J.; Merchenthaler, I.;. Neuroendocrinol. 2000, 21, 95; Ahmed, S. A.; Toxicology 2000, 150, 191.

62. Rajapakse, N.; Silva, E.; KortenKamp, A.; Environ. Hlth. Perspect. 2002, 110, 917; Silva, E.; Rajapakse, N.; KortenKamp, A.; Environ. Sci. Technol. 2002, 36, 1751 